\title{
THE ANALYSIS OF MERGERS AND ACQUISITIONS IN SERBIA AND POLAND IN THE PERIOD 2005 - 2014: A SECTORAL APPROACH
}

Piotr Luty

Wroclaw University of Economics, Wroclaw, Poland

\begin{abstract}
:
The principal purpose of the paper is to analyze the mergers of domestic companies in Serbia during the period 2005 - 2014. The research included mergers with a "positive" (completed transaction) and "negative" outcome (interrupted or unfinished transaction). The methods of statistical analysis and comparative analysis were used herein. Conclusions can be drawn that the global financial crisis had an impact on the number of mergers between business entities. In the analyzed period in Poland, a negative correlation was observed between the mergers of manufacturing companies and the mergers of companies in which the acquirer was a financial company.
\end{abstract}

Keywords:

mergers, acquisitions, accounting.

\section{INTRODUCTION}

Mergers of business units are an essential part of the economic activity of those companies. In this way, the companies can enhance economic activities, expand sales markets, acquire strategic assets, get rid of unprofitable business segments, and carry out restructuring. There are numerous theories attempting to describe the motives for mergers of entities, as well as the effects that appear before and after the merger. Among numerous theories the most noteworthy are those based on "agency costs", which justify a decrease in added value, or even the failure of the merger, as well as the theories that use market efficiency (in this case the market for mergers and acquisitions of companies).

The theory of "agency costs" refers to a situation where there is a conflict of interest between the owners and managerial staff pursuing their own goals. From the owner's perspective, any decision should lead to an increase in the value of the company. On the other hand, increasing the company's profitability may result in an increased insolvency risk, which is in turn negatively perceived by managers. In business mergers, the final decisions are made by the owners on the basis of resolutions of the receiving and acquired companies. However, the complex preparatory phase of the business merger is carried out mainly by the boards of individual companies.

At this stage, there may be a problem of the "principal - agent", which are respectively the owners and board members. Themes in favor of the merger can result from excessive optimism and revaluation of the expected effects both from the acquiring and the acquired companies (Jensen, 2005; Malmendier \& Tate, 2008). Additionally, the merger may stem from a desire to preserve the positions held by the management (Shleifer \& Vishny, 1989; Ravenscraft \& Scherer, 1987; Black, 1989)

Efficient market theory claims that a business merger will take place only in situations where the merger will bring benefits to all parties (both acquiring and acquired companies). Numerous studies concerning mergers suggest that the effects of synergy, both operational and allocative (Weitzel et al.) are the primary motives for the deci-
Correspondence:

Piotr Luty

e-mail:

piotr.luty@ue.wroc.pl 
sion on the merger (Devos et al., 2008; Weston et al., 2004; Mukherjee et al., 2004). In addition, by assuming efficient market mergers and acquisitions, we can conclude that the presence of undervalued, poorly managed companies in the market will result in the emergence of another entity that, as a result of the merger, will produce synergy effects and better use of the companies' resources (Weston et al., 2004).

Regardless of the motives, mergers either take place or companies decide to suspend the integration process. This article aims to analyze business mergers in Serbia and Poland and the success and failure ratio of the merger process. The analysis will include both the acquiring and acquired companies in various industries.

\section{METHODOLOGY AND RESEARCH SAMPLE}

Comparative data of the merging companies is derived from the Thomson Reuters Eikon database. The analysis included mergers and acquisitions of all companies included in the database until the year 2014. Due to the fact that the database was accessed at the end of 2014, not all the mergers that took place in 2014 may be included.

The database included:

- 134 files about the mergers of two companies from Serbia (the acquirer and the acquiree)
- 37 files about mergers where the acquirer was a company from Serbia, and the acquiree was a company from another country

- 326 files on mergers where the acquiree was from Serbia and the acquirer was from another country

Figure 1 presents the number of mergers in the period from 2005 to 2014 (domestic mergers in Serbia).

Figure 2 presents the distribution of domestic mergers of companies in Serbia, which ended with the merger or acquisition of companies. This type of finalized mergers or acquisitions will be referred to as "positive" acquisitions in the article.

Figure 3 presents mergers and acquisitions of domestic companies in Serbia which ultimately were not carried out, or seek a new investor. This type of merger will be referred to herein as a "negative" acquisition.

The division of mergers into "positive” and "negative” is conventional and is used to extract two subgroups from the research sample. It is not the author's intention to verify whether, from the point of view of the acquirers or acquirees, positive acquisition would break the integration process (of the merger or acquisition), or if the mergers had the character of a hostile takeover.

The analysis of mergers will also include national mergers, which took place in Poland during the period 2005-2014.

Figure 1. The number of mergers in Serbia in the period between 2005 and 2014

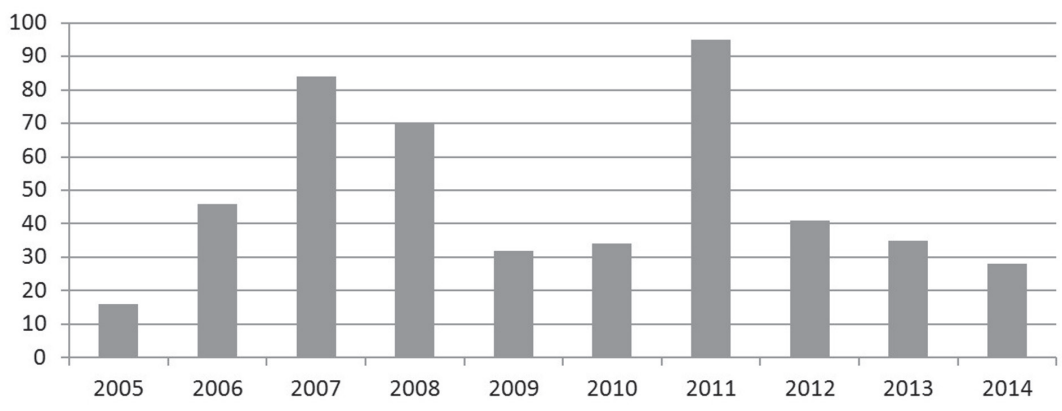

Source: Authors' calculations

Figure 2. "Positive” mergers Serbia-Serbia

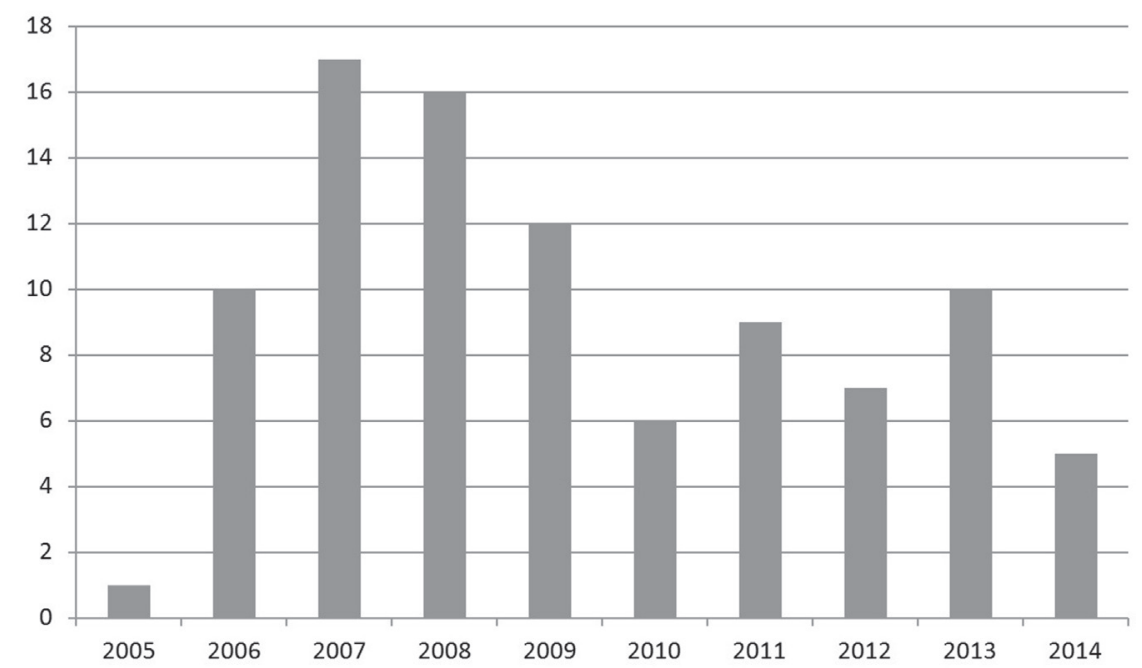


The Reuters database included:

- 2210 files about mergers between Polish companies (the acquirer and the acquiree were both from Poland)

- 224 files about mergers where the acquiring company was Polish and the acquired company was from another country
- 1066 files about mergers where the acquired company was Polish and the acquiring company was from another country

Figure 4 presents the number of merger transactions (positive and negative) for the period 2005-2014.

Figure 5 presents the number of "positively" concluded domestic mergers in Poland.

Figure 3. "Negative" mergers Serbia-Serbia

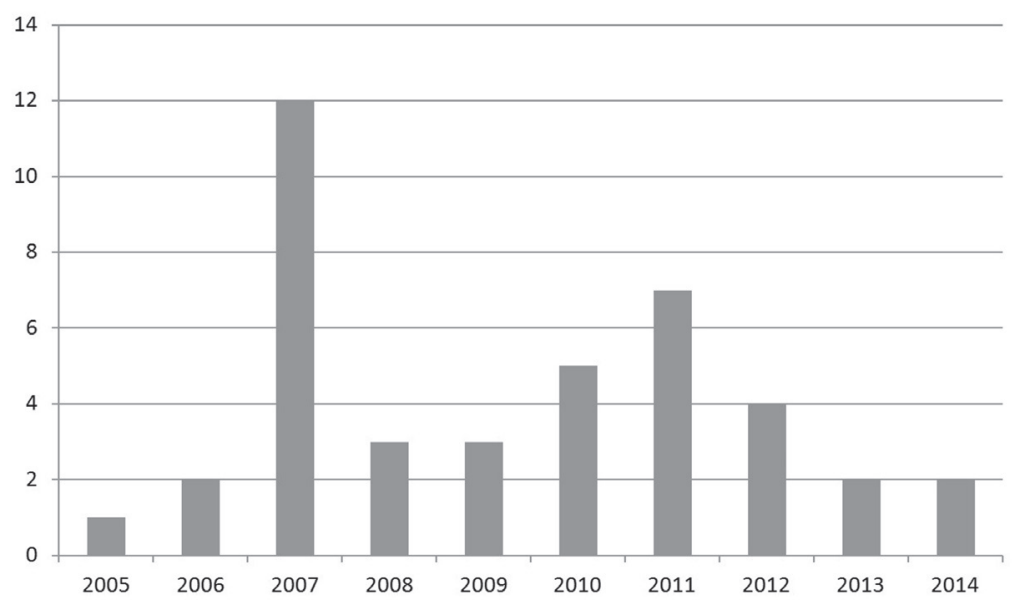

Source: Authors' calculations

Figure 4. The number of mergers in Poland during the period 2005-2014

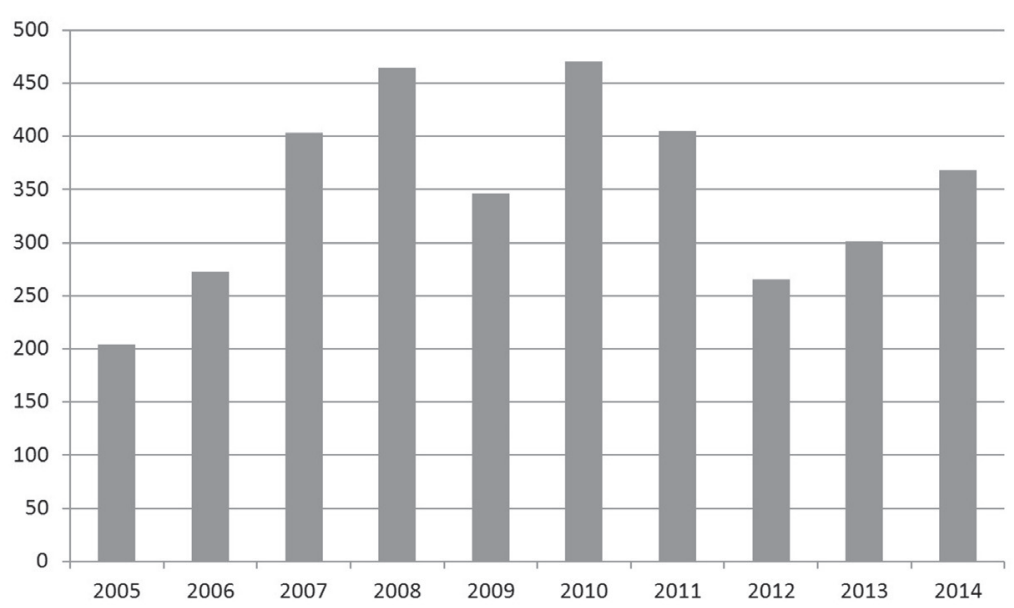

Source: Authors' calculations

Figure 5. "Positive" mergers Poland-Poland

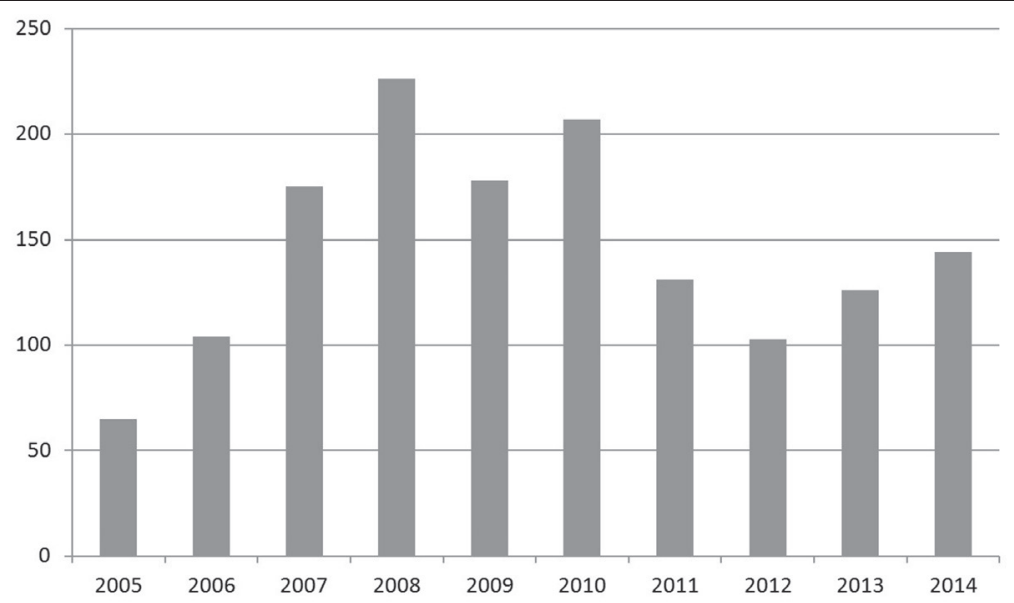


Figure 6 presents the number of "negatively" completed domestic mergers in Poland.

Comparison of the intensity of merger and acquisition processes in Poland and Serbia is presented in Figure 7 and Figure 8 . The charts also indicate the moving average line for the two periods.
Based on the analysis of national mergers in Serbia and Poland, it can be noted that despite different number of transactions in these countries, the shape of the moving average is similar. The key moment is the emergence of the global financial crisis in 2008-2009. In the case of national mergers completed "positively" in 2009, there was a decrease in the

Figure 6. "Negative" mergers Poland-Poland

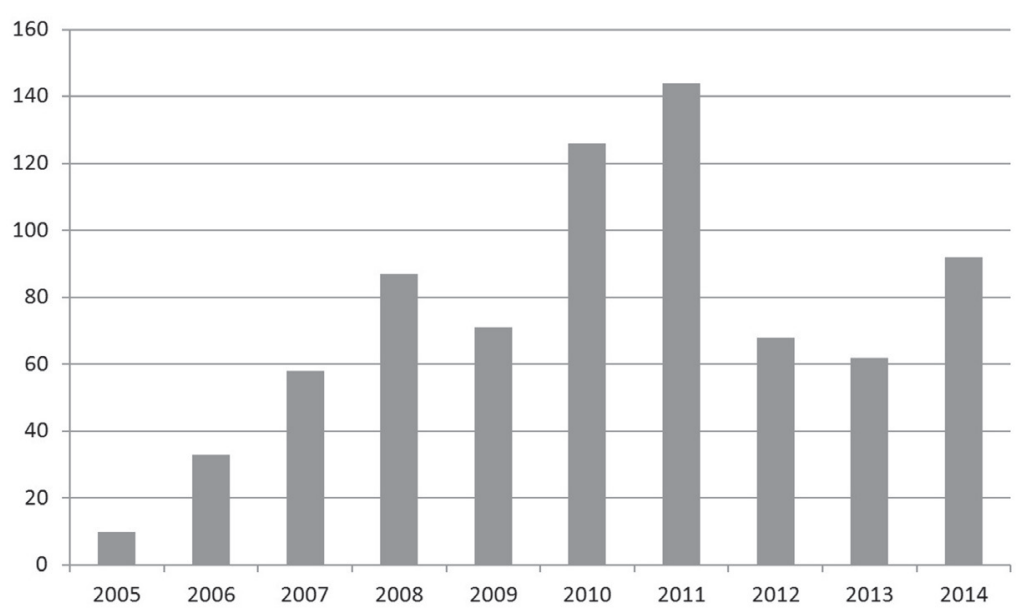

Source: Authors' calculations

Figure 7. Comparison of mergers with positive results in Serbia and Poland

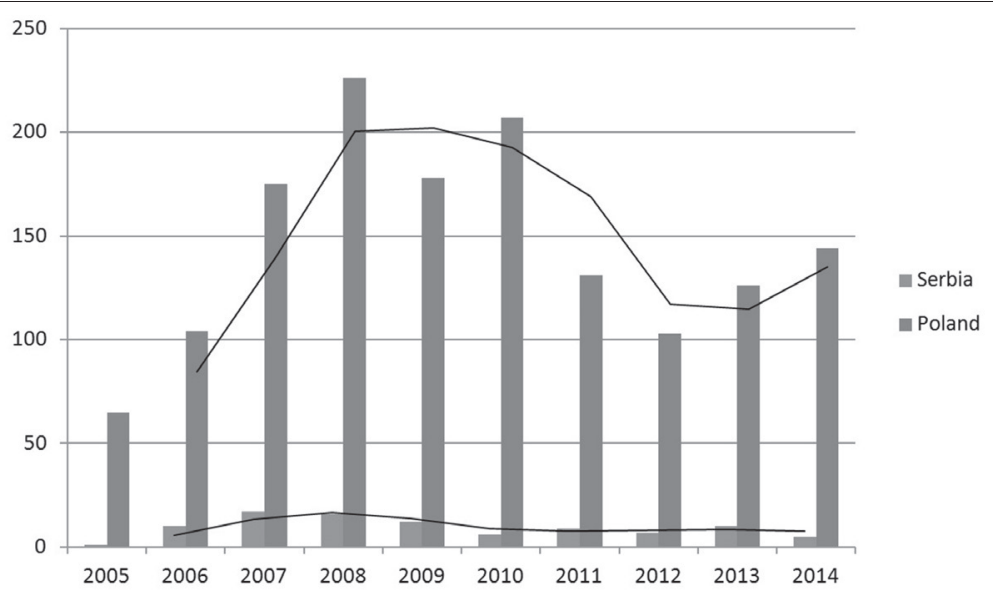

Source: Authors' calculations

Figure 8. Comparison of "negative" mergers in Serbia and Poland

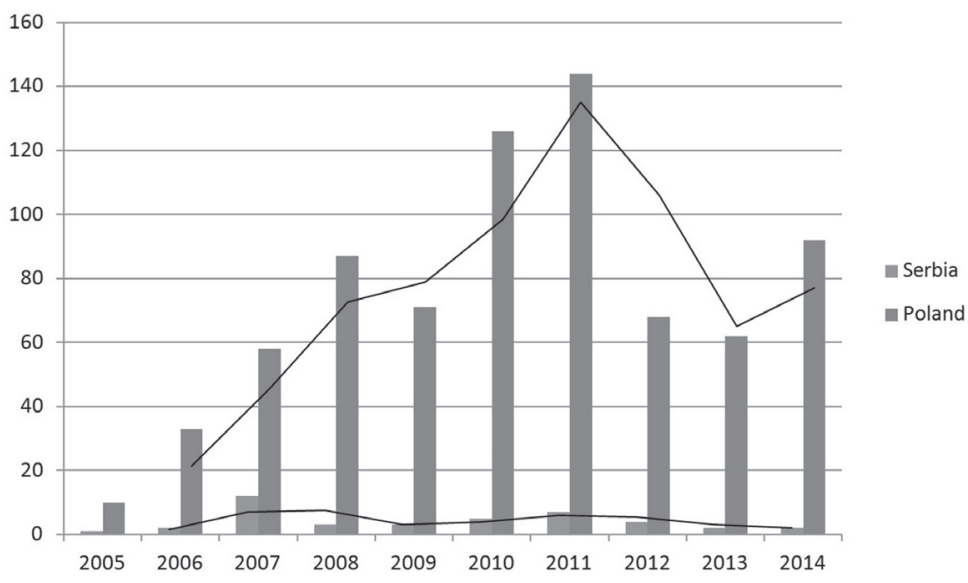


number of mergers, while at the same time the number of 'negative' mergers began to rise.

All companies involved in the merger process have been assigned to the appropriate sector of economic activity. For the purposes of this article, the following sections are distinguished:

- Production

- Services

- Finance

- Trade

- Other

The "Other" category was used in the case when the database did not specify the industry of the acquiring or the acquired company, and when one of the parties was the state or local government institution.

In addition, the merger analysis includes situations where the merger took place, as well as other cases (merger did not happen or a company withdrew from the integration process). There was no analysis of the causes of success or failure of a merger.

The research on Serbian and Polish mergers has been divided into the following stages:

1. The sectoral analysis of domestic mergers in Serbia (merger process completed positively)

2. The sectoral analysis of domestic mergers in Poland (merger process ended positively)
3. The sectoral analysis of domestic mergers in Serbia (merger process completed negatively)

4. The sectoral analysis of domestic mergers in Poland (merger process completed negatively)

\section{RESEARCH RESULTS}

The results will be presented in tables according to the approved research methodology. The tables describing the acquiring and the acquired companies are presented as a percentage of a specific line. In other tables, information about the acquiring companies (in rows) and the acquired companies (in columns) is included.

\section{Sectoral analysis of domestic mergers in Serbia (merger process completed positively)}

Table 1 presents domestic mergers in Serbia from the perspective of the acquiring company. The columns indicate the sectors of economic activity for the acquiring companies.

Based on the data in Table 1, it can be noted that from 2005 to 2010, the share of production companies as the acquirers was increasing, while it began to decrease from 2011. It is also interesting that the largest share in the acquirers was held by financial companies in 2008 (the beginning of the financial crisis), while in the case of production companies, the largest share occurred in 2010 (after the crisis).

Table 1. Mergers Serbia-Serbia - the acquiring companies

\begin{tabular}{cccccccc}
\hline Year & Other & Services & Finance & Energy & Production & Trade & Total \\
\hline 2005 & $0,00 \%$ & $0,00 \%$ & $100,00 \%$ & $0,00 \%$ & $0,00 \%$ & $0,00 \%$ & $100,00 \%$ \\
\hline 2006 & $10,00 \%$ & $0,00 \%$ & $30,00 \%$ & $20,00 \%$ & $40,00 \%$ & $0,00 \%$ & $100,00 \%$ \\
\hline 2007 & $0,00 \%$ & $23,53 \%$ & $23,53 \%$ & $5,88 \%$ & $47,06 \%$ & $0,00 \%$ & $100,00 \%$ \\
\hline 2008 & $0,00 \%$ & $12,50 \%$ & $43,75 \%$ & $0,00 \%$ & $37,50 \%$ & $6,25 \%$ & $100,00 \%$ \\
\hline 2009 & $8,33 \%$ & $33,33 \%$ & $25,00 \%$ & $0,00 \%$ & $16,67 \%$ & $16,67 \%$ & $100,00 \%$ \\
\hline 2010 & $0,00 \%$ & $16,67 \%$ & $0,00 \%$ & $0,00 \%$ & $83,33 \%$ & $0,00 \%$ & $100,00 \%$ \\
\hline 2011 & $0,00 \%$ & $11,11 \%$ & $22,22 \%$ & $0,00 \%$ & $55,56 \%$ & $11,11 \%$ & $100,00 \%$ \\
\hline 2012 & $0,00 \%$ & $42,86 \%$ & $0,00 \%$ & $0,00 \%$ & $42,86 \%$ & $14,29 \%$ & $100,00 \%$ \\
\hline 2013 & $0,00 \%$ & $30,00 \%$ & $30,00 \%$ & $0,00 \%$ & $30,00 \%$ & $10,00 \%$ & $100,00 \%$ \\
\hline 2014 & $0,00 \%$ & $0,00 \%$ & $40,00 \%$ & $0,00 \%$ & $60,00 \%$ & $0,00 \%$ & $100,00 \%$ \\
\hline
\end{tabular}

Source: Authors' calculations

Table 2. Mergers Serbia-Serbia - acquired companies

\begin{tabular}{cccccccc}
\hline Year & Services & Production & Energy & Finance & Trade & Total \\
\hline 2005 & $0,00 \%$ & $100,00 \%$ & $0,00 \%$ & $0,00 \%$ & $0,00 \%$ & $100,00 \%$ \\
\hline 2006 & $10,00 \%$ & $50,00 \%$ & $0,00 \%$ & $20,00 \%$ & $20,00 \%$ & $100,00 \%$ \\
\hline 2007 & $23,53 \%$ & $58,82 \%$ & $0,00 \%$ & $5,88 \%$ & $11,76 \%$ & $100,00 \%$ \\
\hline 2008 & $25,00 \%$ & $43,75 \%$ & $0,00 \%$ & $12,50 \%$ & $18,75 \%$ & $100,00 \%$ \\
\hline 2009 & $25,00 \%$ & $58,33 \%$ & $0,00 \%$ & $0,00 \%$ & $16,67 \%$ & $100,00 \%$ \\
\hline 2010 & $0,00 \%$ & $100,00 \%$ & $0,00 \%$ & $0,00 \%$ & $0,00 \%$ & $100,00 \%$ \\
\hline 2011 & $0,00 \%$ & $66,67 \%$ & $11,11 \%$ & $0,00 \%$ & $22,22 \%$ & $100,00 \%$ \\
\hline 2012 & $28,57 \%$ & $42,86 \%$ & $0,00 \%$ & $14,29 \%$ & $14,29 \%$ & $100,00 \%$ \\
\hline 2013 & $30,00 \%$ & $50,00 \%$ & $10,00 \%$ & $10,00 \%$ & $0,00 \%$ & $100,00 \%$ \\
\hline 2014 & $20,00 \%$ & $80,00 \%$ & $0,00 \%$ & $0,00 \%$ & $0,00 \%$ & $100,00 \%$ \\
\hline
\end{tabular}


Table 2 presents mergers of domestic companies in Serbia from the perspective of the acquired companies. The column shows the sectors of economic activity for the acquirees.

The data in Table 2 show that the largest share of the acquired companies were production companies in the period from 2005 to 2014 . There are three instances when the share of production companies among the acquirees was the largest (these were the years: 2005, 2010 and 2014).

Table 3 presents the percentages of the merging companies for the period from 2005 to 2014 . The columns include sectors of economic activity for the acquirees, while the rows present the acquirers.

On the basis of the data in Table 3 , it can be noted that in the years 2010 and 2011, the largest share in the mergers were those of the production industry (both the acquiring and the acquired companies). During the period 2008 - 2009, there was an increase of mergers in which the acquirer was a company from the financial sector.

Table 3. Mergers Serbia-Serbia - altogether

\begin{tabular}{|c|c|c|c|c|c|}
\hline & Services & Production & Energy & Finance & Trade \\
\hline \multicolumn{6}{|c|}{2005} \\
\hline finance & $0 \%$ & $100 \%$ & $0 \%$ & $0 \%$ & $0 \%$ \\
\hline \multicolumn{6}{|c|}{2006} \\
\hline other & $0 \%$ & $0 \%$ & $0 \%$ & $10 \%$ & $0 \%$ \\
\hline finance & $10 \%$ & $10 \%$ & $0 \%$ & $10 \%$ & $0 \%$ \\
\hline energy & $0 \%$ & $10 \%$ & $0 \%$ & $0 \%$ & $10 \%$ \\
\hline production & $0 \%$ & $30 \%$ & $0 \%$ & $0 \%$ & $10 \%$ \\
\hline \multicolumn{6}{|c|}{2007} \\
\hline services & $12 \%$ & $6 \%$ & $0 \%$ & $0 \%$ & $6 \%$ \\
\hline finance & $6 \%$ & $12 \%$ & $0 \%$ & $6 \%$ & $0 \%$ \\
\hline energy & $0 \%$ & $6 \%$ & $0 \%$ & $0 \%$ & $0 \%$ \\
\hline production & $6 \%$ & $35 \%$ & $0 \%$ & $0 \%$ & $6 \%$ \\
\hline \multicolumn{6}{|c|}{2008} \\
\hline services & $6 \%$ & $0 \%$ & $0 \%$ & $6 \%$ & $0 \%$ \\
\hline finance & $13 \%$ & $19 \%$ & $0 \%$ & $6 \%$ & $6 \%$ \\
\hline production & $6 \%$ & $25 \%$ & $0 \%$ & $0 \%$ & $6 \%$ \\
\hline trade & $0 \%$ & $0 \%$ & $0 \%$ & $0 \%$ & $6 \%$ \\
\hline \multicolumn{6}{|c|}{2009} \\
\hline other & $0 \%$ & $8 \%$ & $0 \%$ & $0 \%$ & $0 \%$ \\
\hline services & $25 \%$ & $8 \%$ & $0 \%$ & $0 \%$ & $0 \%$ \\
\hline finance & $0 \%$ & $25 \%$ & $0 \%$ & $0 \%$ & $0 \%$ \\
\hline production & $0 \%$ & $17 \%$ & $0 \%$ & $0 \%$ & $0 \%$ \\
\hline trade & $0 \%$ & $0 \%$ & $0 \%$ & $0 \%$ & $17 \%$ \\
\hline \multicolumn{6}{|c|}{2010} \\
\hline services & $0 \%$ & $17 \%$ & $0 \%$ & $0 \%$ & $0 \%$ \\
\hline production & $0 \%$ & $83 \%$ & $0 \%$ & $0 \%$ & $0 \%$ \\
\hline \multicolumn{6}{|c|}{2011} \\
\hline services & $0 \%$ & $0 \%$ & $0 \%$ & $0 \%$ & $11 \%$ \\
\hline finance & $0 \%$ & $11 \%$ & $11 \%$ & $0 \%$ & $0 \%$ \\
\hline production & $0 \%$ & $56 \%$ & $0 \%$ & $0 \%$ & $0 \%$ \\
\hline trade & $0 \%$ & $0 \%$ & $0 \%$ & $0 \%$ & $11 \%$ \\
\hline \multicolumn{6}{|c|}{2012} \\
\hline services & $14 \%$ & $29 \%$ & $0 \%$ & $0 \%$ & $0 \%$ \\
\hline production & $14 \%$ & $14 \%$ & $0 \%$ & $0 \%$ & $14 \%$ \\
\hline trade & $0 \%$ & $0 \%$ & $0 \%$ & $14 \%$ & $0 \%$ \\
\hline \multicolumn{6}{|c|}{2013} \\
\hline services & $20 \%$ & $10 \%$ & $0 \%$ & $0 \%$ & $0 \%$ \\
\hline finance & $0 \%$ & $20 \%$ & $10 \%$ & $0 \%$ & $0 \%$ \\
\hline production & $10 \%$ & $20 \%$ & $0 \%$ & $0 \%$ & $0 \%$ \\
\hline trade & $0 \%$ & $0 \%$ & $0 \%$ & $10 \%$ & $0 \%$ \\
\hline \multicolumn{6}{|c|}{2014} \\
\hline finance & $20 \%$ & $20 \%$ & $0 \%$ & $0 \%$ & $0 \%$ \\
\hline production & $0 \%$ & $60 \%$ & $0 \%$ & $0 \%$ & $0 \%$ \\
\hline
\end{tabular}

Source: Authors' calculations 
Sectoral analysis of domestic mergers in Poland (merger process ended positively)

Table 4 presents the shares of the acquiring companies in Poland during the period 2005-2014, divided by sectors of economic activity.

In 2007 (the year prior to the financial crisis), the leading acquiring companies were production companies (50.29\%), whereas financial companies had $16.57 \%$ of share. In the case of financial companies, they reached the smallest percentage in 2007. During the period from 2010 to 2014, the share of financial companies among the acquirers was steadily increasing (from $28.99 \%$ to $58.33 \%$ ), and the shares of production companies decreased (from $37.20 \%$ to $22.22 \%$ ), with the exception of 2012, when they increased to $34.95 \%$.

Table 5 presents the acquired companies in Poland during the period 2005 - 2014, divided by sectors of economic activity.

Among the acquired companies in Poland, the largest share in any year belonged to production companies. In 2009 , both in the group of production and financial companies, local peaks can be observed.

Table 6 shows the percentages of the merging companies (the acquiring and the acquired) in individual years.

Based on the data in Table 6, it can be noted that during the period 2005-2013, most mergers were carried out be- tween the companies of the production industry. The share of merges where the acquirer was a company from the financial sector began to increase in 2013. In addition, it can be noted that in the case of the production and service sector, most mergers took place between the companies of the same sector. In the financial sector, the trend was different. In the mergers where the acquiring company was from the financial sector, the acquired companies usually belonged to the production sector.

\section{Sector analysis of domestic mergers in Serbia (merger processes completed negatively)}

As previously mentioned in the section on methodology and research sample, the process of "negative" mergers is a symbolic term. In this group of events, there are situations concerning lack of an investor, interrupted integration process or revealed "rumour" about the intention to merge. A characteristic feature of these events is their lack of a final merger of business entities.

Table 7 presents the acquiring companies divided by sectors of economic activity.

Based on the data in Table 7, it can be noted that in the case of the most "unsuccessful" mergers in Serbia in the period 2005-2014, the acquirers were the companies from the production sector.

Table 4. Mergers Poland-Poland - the acquiring company

\begin{tabular}{cccccccc}
\hline Year & Production & Services & Finance & Trade & Energy & Other & Total \\
\hline 2005 & $43,08 \%$ & $24,62 \%$ & $21,54 \%$ & $4,62 \%$ & $6,15 \%$ & $0,00 \%$ & $100,00 \%$ \\
\hline 2006 & $43,27 \%$ & $22,12 \%$ & $28,85 \%$ & $2,88 \%$ & $2,88 \%$ & $0,00 \%$ & $100,00 \%$ \\
\hline 2007 & $50,29 \%$ & $24,57 \%$ & $16,57 \%$ & $4,00 \%$ & $4,00 \%$ & $0,57 \%$ & $100,00 \%$ \\
\hline 2008 & $37,61 \%$ & $25,22 \%$ & $28,32 \%$ & $3,54 \%$ & $4,42 \%$ & $0,88 \%$ & $100,00 \%$ \\
\hline 2009 & $30,34 \%$ & $25,84 \%$ & $33,71 \%$ & $2,81 \%$ & $4,49 \%$ & $2,81 \%$ & $100,00 \%$ \\
\hline 2010 & $37,20 \%$ & $23,19 \%$ & $28,99 \%$ & $7,25 \%$ & $2,42 \%$ & $0,97 \%$ & $100,00 \%$ \\
\hline 2011 & $32,06 \%$ & $19,08 \%$ & $34,35 \%$ & $6,11 \%$ & $8,40 \%$ & $0,00 \%$ & $100,00 \%$ \\
\hline 2012 & $34,95 \%$ & $21,36 \%$ & $34,95 \%$ & $2,91 \%$ & $5,83 \%$ & $0,00 \%$ & $100,00 \%$ \\
\hline 2013 & $28,57 \%$ & $26,98 \%$ & $36,51 \%$ & $3,17 \%$ & $4,76 \%$ & $0,00 \%$ & $100,00 \%$ \\
\hline 2014 & $22,22 \%$ & $14,58 \%$ & $58,33 \%$ & $2,08 \%$ & $2,78 \%$ & $0,00 \%$ & $100,00 \%$ \\
\hline
\end{tabular}

Source: Authors' calculations

Table 5. Mergers Poland-Poland - the acquired companies

\begin{tabular}{cccccccc}
\hline Year & Production & energy & Finance & Services & Trade & other & Total \\
\hline 2005 & $36,92 \%$ & $9,23 \%$ & $20,00 \%$ & $27,69 \%$ & $6,15 \%$ & $0,00 \%$ & $100,00 \%$ \\
\hline 2006 & $53,85 \%$ & $2,88 \%$ & $19,23 \%$ & $17,31 \%$ & $6,73 \%$ & $0,00 \%$ & $100,00 \%$ \\
\hline 2007 & $52,57 \%$ & $5,14 \%$ & $7,43 \%$ & $29,71 \%$ & $5,14 \%$ & $0,00 \%$ & $100,00 \%$ \\
\hline 2008 & $47,35 \%$ & $6,64 \%$ & $8,41 \%$ & $30,53 \%$ & $7,08 \%$ & $0,00 \%$ & $100,00 \%$ \\
\hline 2009 & $49,44 \%$ & $5,62 \%$ & $11,24 \%$ & $29,21 \%$ & $4,49 \%$ & $0,00 \%$ & $100,00 \%$ \\
\hline 2010 & $48,31 \%$ & $3,38 \%$ & $7,73 \%$ & $32,85 \%$ & $7,25 \%$ & $0,48 \%$ & $100,00 \%$ \\
\hline 2011 & $43,51 \%$ & $11,45 \%$ & $12,21 \%$ & $25,95 \%$ & $6,87 \%$ & $0,00 \%$ & $100,00 \%$ \\
\hline 2012 & $44,66 \%$ & $8,74 \%$ & $14,56 \%$ & $28,16 \%$ & $3,88 \%$ & $0,00 \%$ & $100,00 \%$ \\
\hline 2013 & $43,65 \%$ & $5,56 \%$ & $15,08 \%$ & $25,40 \%$ & $9,52 \%$ & $0,79 \%$ & $100,00 \%$ \\
\hline 2014 & $43,75 \%$ & $9,03 \%$ & $17,36 \%$ & $29,17 \%$ & $0,69 \%$ & $0,00 \%$ & $100,00 \%$ \\
\hline
\end{tabular}


Table 6. Mergers Poland-Poland - altogether

\begin{tabular}{|c|c|c|c|c|c|c|}
\hline & Production & Energy & Finance & Services & Trade & Other \\
\hline \multicolumn{7}{|c|}{2005} \\
\hline production & $23 \%$ & $3 \%$ & $8 \%$ & $8 \%$ & $2 \%$ & $0 \%$ \\
\hline services & $6 \%$ & $0 \%$ & $2 \%$ & $17 \%$ & $0 \%$ & $0 \%$ \\
\hline finance & $8 \%$ & $0 \%$ & $11 \%$ & $3 \%$ & $0 \%$ & $0 \%$ \\
\hline trade & $0 \%$ & $0 \%$ & $0 \%$ & $0 \%$ & $5 \%$ & $0 \%$ \\
\hline energy & $0 \%$ & $6 \%$ & $0 \%$ & $0 \%$ & $0 \%$ & $0 \%$ \\
\hline \multicolumn{7}{|c|}{2006} \\
\hline production & $36 \%$ & $0 \%$ & $3 \%$ & $2 \%$ & $3 \%$ & $0 \%$ \\
\hline services & $9 \%$ & $0 \%$ & $4 \%$ & $10 \%$ & $0 \%$ & $0 \%$ \\
\hline finance & $9 \%$ & $1 \%$ & $13 \%$ & $6 \%$ & $1 \%$ & $0 \%$ \\
\hline trade & $0 \%$ & $0 \%$ & $0 \%$ & $0 \%$ & $3 \%$ & $0 \%$ \\
\hline energy & $1 \%$ & $2 \%$ & $0 \%$ & $0 \%$ & $0 \%$ & $0 \%$ \\
\hline \multicolumn{7}{|c|}{2007} \\
\hline production & $41 \%$ & $0 \%$ & $1 \%$ & $7 \%$ & $2 \%$ & $0 \%$ \\
\hline services & $6 \%$ & $1 \%$ & $2 \%$ & $15 \%$ & $1 \%$ & $0 \%$ \\
\hline finance & $5 \%$ & $1 \%$ & $5 \%$ & $5 \%$ & $1 \%$ & $0 \%$ \\
\hline trade & $1 \%$ & $0 \%$ & $0 \%$ & $2 \%$ & $2 \%$ & $0 \%$ \\
\hline energy & $0 \%$ & $3 \%$ & $0 \%$ & $1 \%$ & $0 \%$ & $0 \%$ \\
\hline other & $0 \%$ & $0 \%$ & $1 \%$ & $0 \%$ & $0 \%$ & $0 \%$ \\
\hline \multicolumn{7}{|c|}{2008} \\
\hline production & $30 \%$ & $1 \%$ & $1 \%$ & $4 \%$ & $2 \%$ & $0 \%$ \\
\hline services & $5 \%$ & $0 \%$ & $1 \%$ & $18 \%$ & $1 \%$ & $0 \%$ \\
\hline finance & $12 \%$ & $0 \%$ & $5 \%$ & $9 \%$ & $1 \%$ & $0 \%$ \\
\hline trade & $0 \%$ & $0 \%$ & $0 \%$ & $0 \%$ & $3 \%$ & $0 \%$ \\
\hline energy & $0 \%$ & $4 \%$ & $0 \%$ & $0 \%$ & $0 \%$ & $0 \%$ \\
\hline other & $0 \%$ & $0 \%$ & $0 \%$ & $0 \%$ & $0 \%$ & $0 \%$ \\
\hline \multicolumn{7}{|c|}{2009} \\
\hline production & $24 \%$ & $3 \%$ & $1 \%$ & $2 \%$ & $1 \%$ & $0 \%$ \\
\hline services & $4 \%$ & $1 \%$ & $0 \%$ & $20 \%$ & $1 \%$ & $0 \%$ \\
\hline finance & $17 \%$ & $0 \%$ & $10 \%$ & $6 \%$ & $1 \%$ & $0 \%$ \\
\hline trade & $1 \%$ & $0 \%$ & $0 \%$ & $0 \%$ & $2 \%$ & $0 \%$ \\
\hline energy & $2 \%$ & $2 \%$ & $1 \%$ & $0 \%$ & $0 \%$ & $0 \%$ \\
\hline other & $2 \%$ & $0 \%$ & $0 \%$ & $1 \%$ & $0 \%$ & $0 \%$ \\
\hline \multicolumn{7}{|c|}{2010} \\
\hline production & $26 \%$ & $2 \%$ & $0 \%$ & $7 \%$ & $2 \%$ & $0 \%$ \\
\hline services & $4 \%$ & $0 \%$ & $3 \%$ & $16 \%$ & $0 \%$ & $0 \%$ \\
\hline finance & $14 \%$ & $0 \%$ & $4 \%$ & $9 \%$ & $1 \%$ & $0 \%$ \\
\hline trade & $2 \%$ & $0 \%$ & $0 \%$ & $1 \%$ & $4 \%$ & $0 \%$ \\
\hline energy & $1 \%$ & $1 \%$ & $0 \%$ & $0 \%$ & $0 \%$ & $0 \%$ \\
\hline other & $1 \%$ & $0 \%$ & $0 \%$ & $0 \%$ & $0 \%$ & $0 \%$ \\
\hline \multicolumn{7}{|c|}{2011} \\
\hline production & $21 \%$ & $2 \%$ & $2 \%$ & $5 \%$ & $2 \%$ & $0 \%$ \\
\hline services & $6 \%$ & $1 \%$ & $2 \%$ & $9 \%$ & $1 \%$ & $0 \%$ \\
\hline finance & $12 \%$ & $3 \%$ & $8 \%$ & $9 \%$ & $2 \%$ & $0 \%$ \\
\hline trade & $2 \%$ & $0 \%$ & $0 \%$ & $3 \%$ & $2 \%$ & $0 \%$ \\
\hline energy & $2 \%$ & $6 \%$ & $0 \%$ & $0 \%$ & $0 \%$ & $0 \%$ \\
\hline \multicolumn{7}{|c|}{2012} \\
\hline production & $28 \%$ & $1 \%$ & $1 \%$ & $3 \%$ & $2 \%$ & $0 \%$ \\
\hline services & $3 \%$ & $1 \%$ & $1 \%$ & $17 \%$ & $0 \%$ & $0 \%$ \\
\hline finance & $11 \%$ & $3 \%$ & $13 \%$ & $8 \%$ & $1 \%$ & $0 \%$ \\
\hline trade & $1 \%$ & $0 \%$ & $0 \%$ & $1 \%$ & $1 \%$ & $0 \%$ \\
\hline energy & $2 \%$ & $4 \%$ & $0 \%$ & $0 \%$ & $0 \%$ & $0 \%$ \\
\hline \multicolumn{7}{|c|}{2013} \\
\hline production & $19 \%$ & $2 \%$ & $3 \%$ & $2 \%$ & $2 \%$ & $0 \%$ \\
\hline services & $7 \%$ & $0 \%$ & $2 \%$ & $16 \%$ & $2 \%$ & $0 \%$ \\
\hline finance & $17 \%$ & $0 \%$ & $10 \%$ & $7 \%$ & $2 \%$ & $0 \%$ \\
\hline trade & $0 \%$ & $0 \%$ & $0 \%$ & $0 \%$ & $3 \%$ & $0 \%$ \\
\hline energy & $0 \%$ & $4 \%$ & $0 \%$ & $0 \%$ & $0 \%$ & $1 \%$ \\
\hline \multicolumn{7}{|c|}{2014} \\
\hline production & $17 \%$ & $1 \%$ & $1 \%$ & $3 \%$ & $1 \%$ & $0 \%$ \\
\hline services & $5 \%$ & $1 \%$ & $1 \%$ & $8 \%$ & $0 \%$ & $0 \%$ \\
\hline finance & $22 \%$ & $5 \%$ & $14 \%$ & $18 \%$ & $0 \%$ & $0 \%$ \\
\hline trade & $1 \%$ & $0 \%$ & $1 \%$ & $1 \%$ & $0 \%$ & $0 \%$ \\
\hline energy & $0 \%$ & $2 \%$ & $1 \%$ & $0 \%$ & $0 \%$ & $0 \%$ \\
\hline
\end{tabular}

Source: Authors' calculations 
Table 7. Mergers Serbia-Serbia - acquiring companies

\begin{tabular}{cccccccc}
\hline Year & Other & Services & Finance & Energy & Production & Trade & Total \\
\hline 2005 & $0,00 \%$ & $0,00 \%$ & $0,00 \%$ & $0,00 \%$ & $100,00 \%$ & $0,00 \%$ & $100,00 \%$ \\
\hline 2006 & $0,00 \%$ & $0,00 \%$ & $0,00 \%$ & $0,00 \%$ & $100,00 \%$ & $0,00 \%$ & $100,00 \%$ \\
\hline 2007 & $8,33 \%$ & $8,33 \%$ & $16,67 \%$ & $0,00 \%$ & $66,67 \%$ & $0,00 \%$ & $100,00 \%$ \\
\hline 2008 & $0,00 \%$ & $33,33 \%$ & $0,00 \%$ & $0,00 \%$ & $66,67 \%$ & $0,00 \%$ & $100,00 \%$ \\
\hline 2009 & $0,00 \%$ & $33,33 \%$ & $0,00 \%$ & $0,00 \%$ & $66,67 \%$ & $0,00 \%$ & $100,00 \%$ \\
\hline 2010 & $0,00 \%$ & $0,00 \%$ & $20,00 \%$ & $0,00 \%$ & $80,00 \%$ & $0,00 \%$ & $100,00 \%$ \\
\hline 2011 & $0,00 \%$ & $0,00 \%$ & $14,29 \%$ & $14,29 \%$ & $57,14 \%$ & $14,29 \%$ & $100,00 \%$ \\
\hline 2012 & $25,00 \%$ & $0,00 \%$ & $0,00 \%$ & $0,00 \%$ & $75,00 \%$ & $0,00 \%$ & $100,00 \%$ \\
\hline 2013 & $0,00 \%$ & $50,00 \%$ & $0,00 \%$ & $0,00 \%$ & $50,00 \%$ & $0,00 \%$ & $100,00 \%$ \\
\hline 2014 & $0,00 \%$ & $0,00 \%$ & $0,00 \%$ & $0,00 \%$ & $100,00 \%$ & $0,00 \%$ & $100,00 \%$ \\
\hline
\end{tabular}

Source: Authors' calculations

Table 8. Mergers Serbia-Serbia - acquired companies

\begin{tabular}{ccccccc}
\hline Year & Services & Production & Energy & Finance & Trade & Total \\
\hline 2005 & $0,00 \%$ & $100,00 \%$ & $0,00 \%$ & $0,00 \%$ & $0,00 \%$ & $100,00 \%$ \\
\hline 2006 & $0,00 \%$ & $100,00 \%$ & $0,00 \%$ & $0,00 \%$ & $0,00 \%$ & $100,00 \%$ \\
\hline 2007 & $0,00 \%$ & $66,67 \%$ & $0,00 \%$ & $25,00 \%$ & $8,33 \%$ & $100,00 \%$ \\
\hline 2008 & $0,00 \%$ & $100,00 \%$ & $0,00 \%$ & $0,00 \%$ & $0,00 \%$ & $100,00 \%$ \\
\hline 2009 & $33,33 \%$ & $66,67 \%$ & $0,00 \%$ & $0,00 \%$ & $0,00 \%$ & $100,00 \%$ \\
\hline 2010 & $0,00 \%$ & $80,00 \%$ & $0,00 \%$ & $0,00 \%$ & $20,00 \%$ & $100,00 \%$ \\
\hline 2011 & $0,00 \%$ & $57,14 \%$ & $14,29 \%$ & $14,29 \%$ & $14,29 \%$ & $100,00 \%$ \\
\hline 2012 & $25,00 \%$ & $75,00 \%$ & $0,00 \%$ & $0,00 \%$ & $0,00 \%$ & $100,00 \%$ \\
\hline 2013 & $0,00 \%$ & $50,00 \%$ & $0,00 \%$ & $50,00 \%$ & $0,00 \%$ & $100,00 \%$ \\
\hline 2014 & $0,00 \%$ & $100,00 \%$ & $0,00 \%$ & $0,00 \%$ & $0,00 \%$ & $100,00 \%$ \\
\hline
\end{tabular}

Source: Authors' calculations

Table 9. Mergers Serbia-Serbia - altogether

\begin{tabular}{|c|c|c|c|c|c|}
\hline & Services & Production & Energy & Finance & Trade \\
\hline \multicolumn{6}{|c|}{2005} \\
\hline production & $0 \%$ & $100 \%$ & $0 \%$ & $0 \%$ & $0 \%$ \\
\hline \multicolumn{6}{|c|}{2006} \\
\hline production & $0 \%$ & $100 \%$ & $0 \%$ & $0 \%$ & $0 \%$ \\
\hline \multicolumn{6}{|c|}{2007} \\
\hline other & $0 \%$ & $0 \%$ & $0 \%$ & $0 \%$ & $8 \%$ \\
\hline services & $0 \%$ & $8 \%$ & $0 \%$ & $0 \%$ & $0 \%$ \\
\hline finance & $0 \%$ & $0 \%$ & $0 \%$ & $17 \%$ & $0 \%$ \\
\hline production & $0 \%$ & $58 \%$ & $0 \%$ & $8 \%$ & $0 \%$ \\
\hline \multicolumn{6}{|c|}{2008} \\
\hline services & $0 \%$ & $33 \%$ & $0 \%$ & $0 \%$ & $0 \%$ \\
\hline production & $0 \%$ & $67 \%$ & $0 \%$ & $0 \%$ & $0 \%$ \\
\hline \multicolumn{6}{|c|}{2009} \\
\hline services & $33 \%$ & $0 \%$ & $0 \%$ & $0 \%$ & $0 \%$ \\
\hline production & $0 \%$ & $67 \%$ & $0 \%$ & $0 \%$ & $0 \%$ \\
\hline \multicolumn{6}{|c|}{2010} \\
\hline finance & $0 \%$ & $20 \%$ & $0 \%$ & $0 \%$ & $0 \%$ \\
\hline production & $0 \%$ & $60 \%$ & $0 \%$ & $0 \%$ & $20 \%$ \\
\hline \multicolumn{6}{|c|}{2011} \\
\hline finance & $0 \%$ & $0 \%$ & $0 \%$ & $14 \%$ & $0 \%$ \\
\hline energy & $0 \%$ & $0 \%$ & $14 \%$ & $0 \%$ & $0 \%$ \\
\hline production & $0 \%$ & $57 \%$ & $0 \%$ & $0 \%$ & $0 \%$ \\
\hline trade & $0 \%$ & $0 \%$ & $0 \%$ & $0 \%$ & $14 \%$ \\
\hline \multicolumn{6}{|c|}{2012} \\
\hline other & $0 \%$ & $25 \%$ & $0 \%$ & $0 \%$ & $0 \%$ \\
\hline production & $25 \%$ & $50 \%$ & $0 \%$ & $0 \%$ & $0 \%$ \\
\hline \multicolumn{6}{|c|}{2013} \\
\hline services & $0 \%$ & $0 \%$ & $0 \%$ & $50 \%$ & $0 \%$ \\
\hline production & $0 \%$ & $50 \%$ & $0 \%$ & $0 \%$ & $0 \%$ \\
\hline \multicolumn{6}{|c|}{2014} \\
\hline production & $0 \%$ & $100 \%$ & $0 \%$ & $0 \%$ & $0 \%$ \\
\hline
\end{tabular}


Table 8 presents the mergers from the perspective of the acquired companies.

Similarly to the acquiring companies, the acquired ones belonged mainly to the production sector.

Table 9 presents a summary of data for the acquiring companies (in rows) and acquired ones (in columns).

On the basis of the data in Table 9, it can be noted that the largest number of mergers concerned the companies from the production sector.

\section{Sectoral analysis of domestic mergers in Poland (merger process completed negatively)}

Table 10 presents the acquiring companies divided by sectors of economic activity.

It may be noted that the largest part of the acquiring companies came from the production sector. Nevertheless, the financial and service sectors also played an important role. The analysis of the data in Table 10 indicates that in the case of the production sector, the largest share for the acquiring companies was observed in 2007 ( period before the financial crisis). Among these companies, there are two peaks relating to 2007 and 2010. During the period 2010-2013, the share of production companies steadily declined. In turn, the year 2008 (the beginning of the financial crisis) was the moment for the financial sector when its participation in the acquiring companies was the lowest, whereas from 2011 onward it kept an upward trend.
Table 11 presents the division of the acquired companies for the period 2005 - 2014 by sectors of economic activity.

In the case of companies belonging to financial and production sectors, their behaviour was similar to the acquiring companies (Table 10).

Based on the data in Table 12, it can be noted that most "negative" mergers affected companies where both the acquirer and the acquiree come from the same sector of economic activity (production-production; finance-finance, services-services).

\section{CONCLUSION}

Poland and Serbia differ considerably in terms of economic development. In the case of Poland, the integration process in the European Union has had a major significance. Global financial crises have no borders and affect all countries applying the free-market economy. The aim of the article was to analyze domestic mergers taking place in Serbia and Poland with regard to the sectors of economic activities of the merging companies.

Table 6 shows a negative correlation between two groups of "positive" mergers in Poland: the mergers of two production companies and mergers where the acquirer was a financial company (the correlation coefficient was negative with a p-value equal to 0.0221). As regards the mergers between companies in Serbia, no relationship could be observed. This

Table 10. Mergers Poland-Poland - acquiring companies

\begin{tabular}{cccccccc}
\hline Year & Production & Services & Finance & Trade & Energy & Other & Total \\
\hline 2005 & $50,00 \%$ & $10,00 \%$ & $30,00 \%$ & $10,00 \%$ & $0,00 \%$ & $0,00 \%$ & $100,00 \%$ \\
\hline 2006 & $42,42 \%$ & $21,21 \%$ & $27,27 \%$ & $6,06 \%$ & $0,00 \%$ & $3,03 \%$ & $100,00 \%$ \\
\hline 2007 & $51,72 \%$ & $27,59 \%$ & $12,07 \%$ & $0,00 \%$ & $8,62 \%$ & $0,00 \%$ & $100,00 \%$ \\
\hline 2008 & $47,13 \%$ & $33,33 \%$ & $9,20 \%$ & $4,60 \%$ & $5,75 \%$ & $0,00 \%$ & $100,00 \%$ \\
\hline 2009 & $45,07 \%$ & $23,94 \%$ & $18,31 \%$ & $5,63 \%$ & $5,63 \%$ & $1,41 \%$ & $100,00 \%$ \\
\hline 2010 & $49,21 \%$ & $15,08 \%$ & $24,60 \%$ & $5,56 \%$ & $3,97 \%$ & $1,59 \%$ & $100,00 \%$ \\
\hline 2011 & $46,53 \%$ & $26,39 \%$ & $16,67 \%$ & $8,33 \%$ & $1,39 \%$ & $0,69 \%$ & $100,00 \%$ \\
\hline 2012 & $39,71 \%$ & $27,94 \%$ & $19,12 \%$ & $5,88 \%$ & $5,88 \%$ & $1,47 \%$ & $100,00 \%$ \\
\hline 2013 & $29,03 \%$ & $30,65 \%$ & $25,81 \%$ & $9,68 \%$ & $3,23 \%$ & $1,61 \%$ & $100,00 \%$ \\
\hline 2014 & $35,87 \%$ & $25,00 \%$ & $27,17 \%$ & $7,61 \%$ & $4,35 \%$ & $0,00 \%$ & $100,00 \%$ \\
\hline
\end{tabular}

Source: Authors' calculations

Table 11. Merger Poland-Poland - acquired companies

\begin{tabular}{|c|c|c|c|c|c|c|c|}
\hline Year & Production & Energy & Finance & Services & Trade & Other & Total \\
\hline 2005 & $40,00 \%$ & $0,00 \%$ & $30,00 \%$ & $30,00 \%$ & $0,00 \%$ & $0,00 \%$ & $100,00 \%$ \\
\hline 2006 & $42,42 \%$ & $18,18 \%$ & $12,12 \%$ & $15,15 \%$ & $9,09 \%$ & $3,03 \%$ & $100,00 \%$ \\
\hline 2007 & $51,72 \%$ & $10,34 \%$ & $10,34 \%$ & $24,14 \%$ & $3,45 \%$ & $0,00 \%$ & $100,00 \%$ \\
\hline 2008 & $48,28 \%$ & $3,45 \%$ & $2,30 \%$ & $40,23 \%$ & $5,75 \%$ & $0,00 \%$ & $100,00 \%$ \\
\hline 2009 & $46,48 \%$ & $7,04 \%$ & $9,86 \%$ & $28,17 \%$ & $8,45 \%$ & $0,00 \%$ & $100,00 \%$ \\
\hline 2010 & $55,56 \%$ & $7,14 \%$ & $10,32 \%$ & $21,43 \%$ & $5,56 \%$ & $0,00 \%$ & $100,00 \%$ \\
\hline 2011 & $42,36 \%$ & $4,17 \%$ & $13,19 \%$ & $29,17 \%$ & $10,42 \%$ & $0,69 \%$ & $100,00 \%$ \\
\hline 2012 & $45,59 \%$ & $2,94 \%$ & $17,65 \%$ & $25,00 \%$ & $8,82 \%$ & $0,00 \%$ & $100,00 \%$ \\
\hline 2013 & $35,48 \%$ & $4,84 \%$ & $20,97 \%$ & $29,03 \%$ & $9,68 \%$ & $0,00 \%$ & $100,00 \%$ \\
\hline 2014 & $33,70 \%$ & $5,43 \%$ & $15,22 \%$ & $41,30 \%$ & $4,35 \%$ & $0,00 \%$ & $100,00 \%$ \\
\hline
\end{tabular}

Source: Authors' calculations 
Table 12. Merger Poland-Poland - altogether

\begin{tabular}{|c|c|c|c|c|c|c|}
\hline & Production & Energy & Finance & Services & Trade & Other \\
\hline \multicolumn{7}{|c|}{2005} \\
\hline production & $30 \%$ & $0 \%$ & $0 \%$ & $20 \%$ & $0 \%$ & $0 \%$ \\
\hline services & $0 \%$ & $0 \%$ & $0 \%$ & $10 \%$ & $0 \%$ & $0 \%$ \\
\hline finance & $0 \%$ & $0 \%$ & $30 \%$ & $0 \%$ & $0 \%$ & $0 \%$ \\
\hline trade & $10 \%$ & $0 \%$ & $0 \%$ & $0 \%$ & $0 \%$ & $0 \%$ \\
\hline \multicolumn{7}{|c|}{2006} \\
\hline production & $27 \%$ & $6 \%$ & $0 \%$ & $3 \%$ & $3 \%$ & $3 \%$ \\
\hline services & $3 \%$ & $3 \%$ & $0 \%$ & $12 \%$ & $3 \%$ & $0 \%$ \\
\hline finance & $6 \%$ & $9 \%$ & $12 \%$ & $0 \%$ & $0 \%$ & $0 \%$ \\
\hline trade & $3 \%$ & $0 \%$ & $0 \%$ & $0 \%$ & $3 \%$ & $0 \%$ \\
\hline other & $3 \%$ & $0 \%$ & $0 \%$ & $0 \%$ & $0 \%$ & $0 \%$ \\
\hline \multicolumn{7}{|c|}{2007} \\
\hline production & $41 \%$ & $3 \%$ & $0 \%$ & $3 \%$ & $3 \%$ & $0 \%$ \\
\hline services & $3 \%$ & $0 \%$ & $5 \%$ & $19 \%$ & $0 \%$ & $0 \%$ \\
\hline finance & $5 \%$ & $0 \%$ & $5 \%$ & $2 \%$ & $0 \%$ & $0 \%$ \\
\hline energy & $2 \%$ & $7 \%$ & $0 \%$ & $0 \%$ & $0 \%$ & $0 \%$ \\
\hline \multicolumn{7}{|c|}{2008} \\
\hline production & $32 \%$ & $1 \%$ & $0 \%$ & $13 \%$ & $1 \%$ & $0 \%$ \\
\hline services & $7 \%$ & $0 \%$ & $0 \%$ & $25 \%$ & $1 \%$ & $0 \%$ \\
\hline finance & $5 \%$ & $0 \%$ & $2 \%$ & $2 \%$ & $0 \%$ & $0 \%$ \\
\hline trade & $1 \%$ & $0 \%$ & $0 \%$ & $0 \%$ & $3 \%$ & $0 \%$ \\
\hline energy & $3 \%$ & $2 \%$ & $0 \%$ & $0 \%$ & $0 \%$ & $0 \%$ \\
\hline \multicolumn{7}{|c|}{2009} \\
\hline production & $31 \%$ & $1 \%$ & $4 \%$ & $7 \%$ & $1 \%$ & $0 \%$ \\
\hline services & $6 \%$ & $1 \%$ & $0 \%$ & $15 \%$ & $1 \%$ & $0 \%$ \\
\hline finance & $7 \%$ & $0 \%$ & $6 \%$ & $6 \%$ & $0 \%$ & $0 \%$ \\
\hline trade & $0 \%$ & $0 \%$ & $0 \%$ & $0 \%$ & $6 \%$ & $0 \%$ \\
\hline energy & $3 \%$ & $3 \%$ & $0 \%$ & $0 \%$ & $0 \%$ & $0 \%$ \\
\hline other & $0 \%$ & $1 \%$ & $0 \%$ & $0 \%$ & $0 \%$ & $0 \%$ \\
\hline \multicolumn{7}{|c|}{2010} \\
\hline production & $38 \%$ & $2 \%$ & $3 \%$ & $5 \%$ & $1 \%$ & $0 \%$ \\
\hline services & $7 \%$ & $0 \%$ & $0 \%$ & $8 \%$ & $0 \%$ & $0 \%$ \\
\hline finance & $6 \%$ & $2 \%$ & $7 \%$ & $8 \%$ & $2 \%$ & $0 \%$ \\
\hline trade & $3 \%$ & $0 \%$ & $0 \%$ & $0 \%$ & $2 \%$ & $0 \%$ \\
\hline energy & $2 \%$ & $2 \%$ & $0 \%$ & $0 \%$ & $0 \%$ & $0 \%$ \\
\hline other & $0 \%$ & $1 \%$ & $0 \%$ & $1 \%$ & $0 \%$ & $0 \%$ \\
\hline \multicolumn{7}{|c|}{2011} \\
\hline production & $33 \%$ & $3 \%$ & $3 \%$ & $5 \%$ & $3 \%$ & $0 \%$ \\
\hline services & $6 \%$ & $1 \%$ & $1 \%$ & $19 \%$ & $1 \%$ & $0 \%$ \\
\hline finance & $1 \%$ & $0 \%$ & $9 \%$ & $5 \%$ & $1 \%$ & $0 \%$ \\
\hline trade & $2 \%$ & $0 \%$ & $0 \%$ & $1 \%$ & $6 \%$ & $0 \%$ \\
\hline energy & $1 \%$ & $1 \%$ & $0 \%$ & $0 \%$ & $0 \%$ & $0 \%$ \\
\hline other & $0 \%$ & $0 \%$ & $0 \%$ & $0 \%$ & $0 \%$ & $1 \%$ \\
\hline \multicolumn{7}{|c|}{2012} \\
\hline production & $31 \%$ & $0 \%$ & $1 \%$ & $6 \%$ & $1 \%$ & $0 \%$ \\
\hline services & $10 \%$ & $0 \%$ & $1 \%$ & $15 \%$ & $1 \%$ & $0 \%$ \\
\hline finance & $3 \%$ & $0 \%$ & $10 \%$ & $4 \%$ & $1 \%$ & $0 \%$ \\
\hline trade & $0 \%$ & $0 \%$ & $1 \%$ & $0 \%$ & $4 \%$ & $0 \%$ \\
\hline energy & $0 \%$ & $3 \%$ & $3 \%$ & $0 \%$ & $0 \%$ & $0 \%$ \\
\hline other & $1 \%$ & $0 \%$ & $0 \%$ & $0 \%$ & $0 \%$ & $0 \%$ \\
\hline \multicolumn{7}{|c|}{2013} \\
\hline production & $19 \%$ & $2 \%$ & $3 \%$ & $5 \%$ & $0 \%$ & $0 \%$ \\
\hline services & $5 \%$ & $0 \%$ & $5 \%$ & $19 \%$ & $2 \%$ & $0 \%$ \\
\hline finance & $8 \%$ & $2 \%$ & $13 \%$ & $3 \%$ & $0 \%$ & $0 \%$ \\
\hline trade & $2 \%$ & $0 \%$ & $0 \%$ & $0 \%$ & $8 \%$ & $0 \%$ \\
\hline energy & $2 \%$ & $2 \%$ & $0 \%$ & $0 \%$ & $0 \%$ & $0 \%$ \\
\hline other & $0 \%$ & $0 \%$ & $0 \%$ & $2 \%$ & $0 \%$ & $0 \%$ \\
\hline \multicolumn{7}{|c|}{2014} \\
\hline production & $22 \%$ & $0 \%$ & $4 \%$ & $9 \%$ & $1 \%$ & $0 \%$ \\
\hline services & $4 \%$ & $0 \%$ & $0 \%$ & $21 \%$ & $0 \%$ & $0 \%$ \\
\hline finance & $8 \%$ & $1 \%$ & $11 \%$ & $8 \%$ & $0 \%$ & $0 \%$ \\
\hline trade & $0 \%$ & $0 \%$ & $0 \%$ & $4 \%$ & $3 \%$ & $0 \%$ \\
\hline energy & $0 \%$ & $4 \%$ & $0 \%$ & $0 \%$ & $0 \%$ & $0 \%$ \\
\hline
\end{tabular}


means that the activity of investment companies in Poland inhibited the merger of the production industry.

In the case of mergers which were not "positively" concluded, there was no statistically significant correlation between the mergers of two production companies or mergers where the acquirer was a financial company.

The analysis of the number of mergers in Serbia and Poland indicates that the financial crisis that took place in 2008 and 2009 caused a decline in the number of mergers. Based on data in Table 1 and Table 4, it can be noted that during the financial crisis, the share of mergers where the acquirer was a financial company was increasing (Serbia in 2008 43.75\%, Poland in 2009 - 33.71\%).

\section{REFERENCES}

Black, B. (1989). Bidder Overpayment in Takeovers. Stanford Law Review, 41(3), 597-660.

Devos, E., Kadapakkam, P.R., \& Krishnamurthy, S. (2008). How Do Mergers Create Value? A Comparison of Taxes, Market Power, and Efficiency Improvements as Explanations for Synergies, Review of Financial Studies.
Jensen, M.C. (2005). Agency Costs of Overvalued Equity. Financial Management, 34(1).

Malmendier, U., \& Tate, G. (2008). Who makes acquisitions? CEO overconfidence and the market's reaction. Journal of Financial Economics, 89, 20-43.

Mukherjee, T.K., Kiymaz, H., \& Baker, H.K. (2004). Merger Motives and Target Valuation: A Survey of Evidence from CFO's. Journal of Applied Finance, 14, 7-24.

Ravenscraft, D.J., \& Scherer, F.M. (1987). Mergers, Sell-offs and Economic Efficiency. Washington, DC: The Brookings Institution.

Shleifer, A., \& Vishny, R.W. (1989). Management Entrenchment: The Case of Manager-specific Investments. Journal of Financial Economics, 25(1), 123-139.

Weitzel U., \& McCarthy, K.J. (2011). Theory and evidence on mergers and acquisitions by Small and Medium Enterprises, Tjalling C. Koopmans Research Institute, Discussion Paper Series nr: 09-21

Weston, F.J., Mitchell, M.L., \& Mulherin, H.J. (2004). Takeovers, Restructuring and Corporate Governance. Upple Saddle River, New Jersey: Pearson Prentice Hall.

\section{ANALIZA SPAJANJA I PREUZIMANJA PREDUZEĆA U SRBIJI I POLJSKOJ U PERIODU OD 2005. DO 2014. GODINE: SEKTORSKI PRISTUP}

\section{Apstrakt:}

Cilj rada jeste da ispita spajanja domaćih kompanija u Srbiji u periodu od 2005. do 2014. godine. Istraživanje je obuhvatilo spajanja sa "pozitivnim" ishodom (završene transakcije) i "negativnim" ishodom (prekinute ili nedovršene transakcije). Korišćene su metode statističke analize i komparativne analize. $\mathrm{Na}$ osnovu sprovedene analize može se izvesti zaključak da je globalna finansijska kriza uticala na broj spajanja između privrednih subjekata. Tokom posmatranog perioda u Poljskoj, primećena je negativna korelacija između spajanja proizvodnih kompanija i spajanja preduzeća u kojima je sticalac finansijska institucija.
Ključne reči:

spajanje, akvizicije, računovodstvo. 